\title{
Study of interleukin 33 in rheumatoid arthritis versus osteoarthritis patients
}

\author{
Aliaa M. Abd El-Aziz Farag ${ }^{a}$, Nagat M. El-Gazzarb, Marwa A.Abo El Hawab, \\ Mohamed A.Saad Attiac
}

\author{
aDepartment of Physical Medicine, \\ Rheumatology and Rehabilitation, ${ }^{b}$ Department \\ of Physical Medicine, Rheumatology and \\ Rehabilitation, 'Department of Clinical \\ Pathology, Faculty of Medicine, Tanta \\ University, Tanta, Egypt \\ Correspondence to Marwa A. Abo El Hawa, \\ MD, 33 El-Motasem Street, Tanta, 3111, Egypt. \\ Tel: +20 100182 7935; \\ E-mail: marwaaboelhawa@gmail.com
}

Received 1 February 2017

Accepted 7 March 2017

Egyptian Rheumatology \& Rehabilitation 2017, 44:159-163

\begin{abstract}
Aim of the work
To compare the expression of serum interleukin 33 (IL33), a new member of the interleukin1 (IL-1) cytokine family, in rheumatoid arthritis (RA) versus osteoarthritis $(\mathrm{OA})$ patients and to correlate it with clinical, laboratory and radiographic parameters.

\section{Subjects and methods}

20 RA and 20 primary knee OA patients. The levels of serum IL-33 were measured by enzyme-linked immunosorbent assay (ELISA) while anticyclic citrullinated peptide (Anti-CCP), rheumatoid factor (RF), erythrocyte sedimentation rate (ESR) and C-reactive protein (CRP) were measured by standard laboratory techniques. Plain $\mathrm{x}$-ray of both hands and wrists were evaluated using the modified Larsen score 1995 (MLS) in RA patients. Knee OA grading was performed according to the KellgrenLawrence classification. The correlation of IL-33 level with clinical, laboratory and radiological data of RA and OA was analyzed.

Results

Serum IL-33 level was significantly higher in RA than in OA patients $(P<0.001)$. This level was positively correlated with disease duration, clinical and laboratory markers of disease activity, impaired functional status and radiographic severity in RA while not in OA patients.

\section{Conclusions}

These findings support that IL-33 could have an essential proinflammatory role in the pathogenesis of RA and that IL-33 level may be a monitor of disease activity and severity. IL-33 may become therapeutic target for RA.
\end{abstract}

\section{Keywords:}

interleukin-33, osteoarthritis, rheumatoid arthritis

Egypt Rheumatol Rehabil 44:159-163

(C) 2017 Egyptian Society for Rheumatology and Rehabilitation

1110-161X

\section{Introduction}

Rheumatoid arthritis (RA) is a chronic systemic inflammatory disease of unknown etiology that primarily involves the joints but can also cause multiple extra-articular manifestations. $\mathrm{RA}$ is the most common autoimmune disease. It is characterized by synovitis and autoantibody formation. The hallmark feature of the disease is persistent symmetric polyarthritis that affects the hands, wrists and feet, although almost all diarthrodial joints may become involved. The severity of RA may fluctuate over time, but chronic RA most commonly results in the progressive development of various degrees of joint destruction, deformity, significant decline in functional status and premature death [1].

Osteoarthritis (OA) is a common condition associated with pain and irreversible progressive joint damage that undermines the quality of life. This multifactorial disease is characterized by destruction of the joint cartilage, subchondral bone thickening and osteophyte formation [2].

Interleukin 33 (IL-33) is a newly found cytokine of the IL-1 family, which also includes IL-1 $\beta$ and IL-18. It is involved in the RA inflammatory status through the IL-1 receptor-related protein sequential control of Toll-like receptor (ST2) [3]. Anti-ST2 antibody that blocks IL-33 signalling could attenuate the severity of experimental arthritis. These studies suggest that IL-33 contributes to the pathogenesis of joint inflammation and destruction. Synovial fibroblasts are believed to be one of the main sources of IL-33 in RA, producing huge amounts of IL-33 in

This is an open access article distributed under the terms of the Creative Commons Attribution-NonCommercial-ShareAlike 3.0 License, which allows others to remix, tweak, and build upon the work noncommercially, as long as the author is credited and the new creations are licensed under the identical terms. 
the presence of tumor necrosis factor- $\alpha$ stimulation in vitro [4]. IL-33 locates mainly in the nucleus and may regulate the expression of some genes [5]. The role of IL-33 in bone metabolism and remodelling has been studied with conflicting results [6].

\section{Patients and methods \\ Study population}

This study included 20 RA patients and 20 primary knee OA patients. Informed consent was taken from all participants in the study. The study was approved by the ethics committee of the Faculty of Medicine Tanta University with approval code 2600/06/14. All patients were selected from the Physical Medicine, Rheumatology and Rehabilitation Clinics, Tanta University Hospitals.

(1) RA patients were diagnosed according to the American College of Rheumatology/European League Against Rheumatism 2010 Criteria for RA [7].

(2) Primary knee OA patients of either sex were diagnosed according to the clinical criteria developed by American College of Rheumatology for the diagnosis of OAs of the knee [8].

\section{Exclusion criteria}

RA and OA patients who were suffering from the following diseases were excluded from the study: inflammatory skin disorders, inflammatory bowel disease, asthma, cancer, cardiovascular diseases, secondary OA, type 2 diabetes and liver fibrosis (chronic hepatitis $\mathrm{C}$ and chronic hepatitis $\mathrm{B}$ ).

\section{Clinical assessment}

Assessment of rheumatoid factor patients

(1) Morning stiffness: Its duration was assessed in minutes.

(2) Assessment of activity using Disease Activity Score (DAS28) [9].

(3) Functional assessment using Modified Health Assessment Questionnaire [10].

\section{Assessment of osteoarthritis patients}

(1) Pain was assessed using Visual Analogue Scale [11].

(2) Modified Ritchie Articular Index [12].

(3) Morning stiffness: Its duration was assessed in minutes.

(4) Functional assessment was carried out using Lequesne's Algo functional Index [13].
Laboratory assessment of total serum interleukin $\mathbf{3 3}$ level Venous blood samples were collected from all participants with sterile disposable syringes in a sterile tube, and samples were centrifuged at $1000 \mathrm{~g}$ for $15 \mathrm{~min}$ for serum separation with a dry clean Pasteur pipette. The serum samples were frozen at $-70^{\circ} \mathrm{C}$ until used for assay of total serum IL-33 level using ELISA technique [14].

\section{Radiographic assessment}

Radiological assessment of rheumatoid factor patients

Plain x-ray of both hands and wrists was obtained and radiological assessment was carried out using the modified Larsen score 1995 [15].

\section{Radiological assessment of osteoarthritis patients}

OA severity was determined using weight-bearing anteroposterior radiographs of both knees. It was evaluated according to the Kellgren and Lawrence (KL) grading system [16].

\section{Statistical analysis [17]}

Statistical analysis was carried out using the statistical package for social sciences (SPSS) software, version 16.0 for Windows (SPSS Inc., Chicago, Illinois, USA). Demographic data between patients were compared using the $\chi^{2}$ and unpaired Student's $t$-tests when appropriate. Pearson's correlation coefficient $(r)$ was used to determine the correlation between concentrations of IL-33 in plasma and clinical and radiographic parameters. Data are expressed as a mean \pm SD. $P$ values of less than 0.05 were considered statistically significant for differences and correlation.

\section{Results}

There was no statistically significant difference in demographic data between RA and OA patients, but there was a significant difference between RA and OA patients as regards erythrocyte sedimentation rate (ESR) first hour, C-reactive protein (CRP), haemoglobin levels, rheumatoid factor titre, anticyclic citrullinated peptide (CCP) titre and total serum IL-33 levels, as demonstrated in Table 1.

Serum IL-33 levels were not correlated with demographic data (age and sex) in studied RA patients. However, there was a significant correlation between serum IL-33 levels and disease duration, morning stiffness, number of tender joints, number of swollen joints, Visual Analogue Scale of pain, DAS28 score, Modified Health Assessment Questionnaire, ESR first hour, 
Table 1 Demographic and laboratory data in rheumatoid arthritis and osteoarthritis patients

\begin{tabular}{lcc}
\hline & RA patients $(n=20)$ & OA patients $(n=20)$ \\
\hline Demographic data & & $50.05 \pm 5.37$ \\
Age & $45.7 \pm 11.9$ & $2 / 18$ \\
Sex (male/female) & $2 / 18$ & 0.148 \\
Laboratory & & 0.165 \\
Serum IL-33 level (pg/ml) & $44.63 \pm 27.4$ & $3.78 \pm 2.13$ \\
ESR (first hour) (mm/h) & $39.7 \pm 24.07$ & $11 \pm 3.84$ \\
CRP (mg/dl) & $25.5 \pm 12.1$ & $4.8 \pm 1.54$ \\
Haemoglobin levels (g/dl) & $10.8 \pm 1.26$ & $11.96 \pm 1.06$ \\
RF titre (IU) & $60.3 \pm 50.2$ & $<.7 \pm 6.6$ \\
Anti-CCP titre (IU) & $95.6 \pm 56.4$ & $<0.001^{*}$ \\
\hline
\end{tabular}

CCP, cyclic citrullinated peptide; CRP, C-reactive protein; ESR, erythrocyte sedimentation rate; IL, interleukin; OA, osteoarthritis; RA, rheumatoid arthritis; RF, Rheumatoid factor. * means significant difference between the 2 groups $P<0.05$.

Table 2 Correlation between serum interleukin 33 levels and demographic, clinical, laboratory and radiological data in rheumatoid arthritis

\begin{tabular}{|c|c|c|}
\hline & \multicolumn{2}{|c|}{ Serum IL-33 } \\
\hline & $R$ & $P$ \\
\hline Age & 0.119 & 0.617 \\
\hline Sex & 0.196 & 0.409 \\
\hline Disease duration (years) & 0.51 & $0.02^{*}$ \\
\hline Morning stiffness (min) & 0.83 & $0.001^{*}$ \\
\hline Number of tender joints & 0.79 & $<0.001^{*}$ \\
\hline Number of swollen joints & 0.85 & $0.001^{*}$ \\
\hline VAS of pain & 0.58 & $0.007^{*}$ \\
\hline DAS28 score & 0.8 & $<0.001^{*}$ \\
\hline $\mathrm{MHAQ}$ & 0.59 & $0.006^{*}$ \\
\hline ESR (first hour) (mm/h) & 0.8 & $<0.001^{*}$ \\
\hline CRP (mg/dl) & 0.72 & $0.001^{*}$ \\
\hline Haemoglobin levels (g/dl) & -0.06 & 0.79 \\
\hline Rheumatoid factor (IU) & 0.78 & $0.001^{*}$ \\
\hline Anti-CCP (IU) & 0.53 & $0.016^{\star}$ \\
\hline Modified Larsen grades & 0.61 & $0.004^{*}$ \\
\hline
\end{tabular}

CCP, cyclic citrullinated peptide; CRP, C-reactive protein; DAS, Disease Activity Score; ESR, erythrocyte sedimentation rate; IL, interleukin; MHAQ, Modified Health Assessment Questionnaire; VAS, Visual Analogue Scale.

$\mathrm{CRP}$, rheumatoid factor titer, anti-CCP titer and modified Larsen score, whereas there was no significant correlation between serum IL-33 levels and haemoglobin level in RA patients studied, as demonstrated in Table 2 .

Serum IL-33 levels were not correlated with demographic data (age and sex), clinical data, Lequesne Algo functional index, ESR first hour, $\mathrm{CRP}$, rheumatoid factor titre, anti-CCP titre and $\mathrm{KL}$ grades in studied OA patients, as demonstrated in Table 3.

\section{Discussion}

IL-33 possesses dual roles both as a traditional extracellular cytokine and as an intracellular nuclear factor with transcriptional regulatory properties [5].
Table 3 Correlation between serum interleukin 33 levels and demographic, clinical, laboratory and radiological data in osteoarthritis

\begin{tabular}{lcc}
\hline & \multicolumn{2}{c}{ Serum IL-33 } \\
\cline { 2 - 3 } & $R$ & $P$ \\
\hline Age & 0.286 & 0.221 \\
Sex & 0.196 & 0.41 \\
Duration of illness (years) & 0.075 & 0.752 \\
Morning stiffness (min) & -0.055 & 0.82 \\
Tenderness & 0.093 & 0.69 \\
VAS of pain & 0.14 & 0.56 \\
Lequesne Algo functional index (range: 0-24) & 0.078 & 0.74 \\
ESR (mm/h) & -0.037 & 0.88 \\
CRP (mg/dl) & -0.303 & 0.19 \\
Rheumatoid factor (IU) & 0.15 & 0.51 \\
Anti-CCP (IU) & 0.12 & 0.56 \\
KL grades & 0.34 & 0.15 \\
\hline
\end{tabular}

CCP, cyclic citrullinated peptide; CRP, C-reactive protein; ESR, erythrocyte sedimentation rate; IL, interleukin; KL, Kellgren and Lawrence; VAS, Visual Analogue Scale.

IL-33 acts through the Toll/IL-1 receptor domain of IL-1 receptor accessory protein, which is shared by other IL-1 family members, to transduce the IL-33/ST2 signal. The IL-33/ST2 pathway may contribute to the pathogenesis of joint inflammation and destruction [3].

Our study demonstrated that the serum IL-33 levels in RA patients were significantly higher than that in $\mathrm{OA}$ patients. These results are in agreement with Xiangyang et al. [18], Talabot-Ayer et al. [19], Matsuyama et al. [20], Tang et al. [21], Mu et al. [22] and Ali et al. [23], who found that serum IL-33 levels were significantly higher in RA than in OA. This might point to differences in disease mechanism involved in these arthropathy pathogenesis, suggesting that induction of IL-33 expression by fibroblasts might be commonly observed in inflamed or damaged tissues.

However, Hong et al. [24] had found that serum level of IL-33 was higher in patients with RA as well as OA; 
these results support the belief that $\mathrm{OA}$ is an inflammatory disease. Although OA is still generally considered a degenerative disorder, the development and progression of $\mathrm{OA}$ are now believed to involve inflammation even in the early stages of the disease. Secreted inflammatory factors such as proinflammatory cytokines are critical mediators of the disturbed metabolism and enhanced catabolism of joint tissue involved in $\mathrm{OA}$.

In agreement with our observations, Matsuyama et al. [20], Tang et al. [21], Ali et al. [23] and Hong et al. [24] found that serum IL-33 was positively correlated with RA disease activity. Xiangyang et al. [18], Tang et al. [21] and $\mathrm{Mu}$ et al. [22] found a significant positive correlation between IL-33 and RA-associated autoantibodies, which supported the hypothesis that IL-33 plays an important role in the pathogenesis of RA and that IL-33 may contribute to abnormal B-cell autoimmunity and antibody overproduction by inducing mast cell activation. Both human and animal studies have shown that extracellular IL-33 could stimulate the maturation and activation of mast cells. Mast cells have been confirmed to be a cellular link between autoantibodies and inflammatory arthritis, giving new evidence on the involvement of IL-33 in abnormal humoral immunity with profound proinflammatory effect and may help in understanding the complex issue of autoimmunity in RA; thus, IL-33 is considered a risk factor for poor prognosis in RA [25].

$\mathrm{Xu}$ et al. [26] showed that administration of IL-33 invivo exacerbates experimental arthritis and elevates the production of proinflammatory cytokines and anticollagen antibodies. Therefore, IL-33 contributes to the antibody response and the severity of inflammation in a serum-induced arthritis mouse model that is mast cell-dependent. However, against our observations, Xiangyang et al. [20] found a nonsignificant correlation between IL-33 and disease activity in RA, which was scored with the DAS28-CRP.

However, in our OA patients there was a nonsignificant correlation between clinical, laboratory and radiological data (KL grades) with total serum IL-33 levels $(P>0.05)$. Our results are in agreement with the results of Xiangyang et al. [18], Tang et al. [21] and Ali et al. [23].

Our study also showed that the serum IL-33 levels were significantly higher in erosive RA patients when compared with nonerosive RA patients $(P<0.05)$. This observation provides further evidence that
IL-33 may be a mediator of joint damage in RA patients and an indicator for disease severity. In agreement with our observation, Xiangyang et al. [18] found significant correlation between serum IL-33 levels and radiographic joint damage in RA patients (evaluated by modified Sharp Score). This finding supported the hypothesis that IL-33 may partly contribute to the bone erosion in RA patients which demonstrate that the mechanism by which IL-33 plays a destructive role in joints of RA patients is through activation of the transcription factors NF-kB induction of IL-6 in RA synovial fibroblasts which are responsible for cartilage destruction and joint damage as well as IL-6 and tumor necrosis factor- $\alpha$ in monocytes of these patients.

\section{Conclusion}

The serum level of IL-33 was significantly increased in RA patients than in OA patients. This level was positively correlated with disease activity, disease duration, bone erosions, joint damage, and impaired functional status in RA patients, whereas IL-33 level was correlated neither with clinical and laboratory findings nor with radiological findings in OA patients.

These findings suggest that IL-33 could be a useful biochemical parameter to reflect disease activity and severity in RA. Further studies are needed to explore the role of IL-33 in the pathogenesis, disease activity, bone erosions and joint damage, and the possibility of being a novel therapeutic target for RA, as well as to explore its role in $\mathrm{OA}$ and other autoimmune and inflammatory diseases.

\section{Financial support and sponsorship \\ Nil.}

\section{Conflicts of interest}

There are no conflicts of interest.

\section{References}

1 Khurana R, Berney SM. Clinical aspects of rheumatoid arthritis. Pathophysiology 2015; 12:153-165.

2 Pelletier JP, Martel J. Therapeutic targets in osteoarthritis: from today to tomorrow with new imaging technology. Ann Rheum Dis 2003; 62:79-82.

3 Schmitz J, Owyang A, Oldham E, Song Y, Murphy E, McClanahan TK, et al. IL-33, an interleukin-1-like cytokine that signals via the IL-1 receptor-related protein ST2 and induces T helper type 2-associated cytokines. Immunity 2005; 23:479-490.

4 Palmer G, Talabot-Ayer D, Lamacchia C, Toy D, Seemayer CA, Viatte S, etal. Inhibition of interleukin-33 signaling attenuates the severity of experimental arthritis. Arthritis Rheum 2009; 60:738-749.

5 Kakkar R, Lee RT. The IL-33/ST2 pathway: therapeutic target and novel biomarker. Nat Rev Drug Discov 2008; 7:827-840. 
6 Miller AM. Role of IL-33 in inflammation and disease. J Inflamm 2011; 8:22

7 Aletaha D, Neogi T, Silman AJ, Funovits J, Felson DT, Bingham CO 3rd, et al. The 2010 Rheumatoid Arthritis Classification Criteria: an American College of Rheumatology/European League Against Rheumatism Collaborative Initiative. Arthritis Rheum 2010; 62:2569-2581.

8 Altman R, Asch E, Bloch D, et al. The American College of Rheumatology criteria for the classification and reporting of osteoarthritis of the knee. Arthritis Rheum 1986; 29:1039-1049.

9 Prevoo ML, Hof MA, Kuper HH. Modified disease activity scores that include twenty eight- joint counts. Development and validation in a prospective longitudinal study of patients with rheumatoid arthritis. Arth Rheum 1995; 38:44-48.

10 Maska L, Anderson J, Michaud K. Measures of functional status and quality of life in rheumatoid arthritis. Arthritis Care Res 2011; 63:S4-S13.

11 Hawker GA, Main S, Kendzerska T, French M. Visual Analogue Scales (VAS) for pain. Measures of adult pain. Arthritis Care Res 2011; 63 S240-S252.

12 Doyle D, Dieppe $\mathrm{P}$, Jane S, Husskison E. An articular index for the assessment of osteoarthritis. Ann Rheum Dis 1981; 40:75-78.

13 Lequesne MG, Maheu E. Clinical and radiological evaluation of hip, knee and hand osteoarthritis. Aging Clin Exp Res 2003; 15: 380-390.

14 eBioscience Human IL-33 Platinum ELISA BMS2048/BMS2048TEN for research use only. http://www.ebioscience.com/human-il-33-platinumelisa-kit.htm; 2015.

15 Larsen A. How to apply Larsen score in evaluating radiographs of rheumatoid arthritis in long term studies? J Rheumatol 1995; 22:1974-1975.

16 Brandt KD, Fife RS, Braunstein EM, Katz B. Radiographic grading of the severity of knee osteoarthritis: relation of the Kellgren and Lawrence grade to a grade based on joint space narrowing, and correlation with arthroscopic evidence of articular cartilage degeneration. Arthritis Rheum 1991; 34 1381-1386
17 Dawson-Saunders B, Trapp R. Basic and clinical biostatistics. 2nd ed. New York: Lange Medical Book: Prentice-Hall International Inc.; 1994.

18 Xiangyang Z, Lutian Y, Lin Z, Liping X, Hui S, Jing L. Increased levels of interleukin-33 associated with bone erosion and interstitial lung diseases in patients with rheumatoid arthritis. Cytokine 2012; 58:6-9.

19 Talabot-Ayer D, McKee T, Gindre P, Bas S, Baeten DL, Gabay C, et al. Distinct serum and synovial fluid interleukin (IL)-33 levels in rheumatoid arthritis, psoriatic arthritis and osteoarthritis. Joint Bone Spine 2012; 79:32-37.

20 Matsuyama $\mathrm{Y}$, Okazaki $\mathrm{H}$, Tamemoto $\mathrm{H}$, Kimura $\mathrm{H}$, Kamata $\mathrm{Y}$, Nagatani $\mathrm{K}$, et al. Increased levels of interleukin 33 in sera and synovial fluid from patients with active rheumatoid arthritis. J Rheumatol 2010; 37:18-25.

21 Tang S, Huang H, Hu F, Zhou W, Guo J, Jiang H, et al. Increased IL-33 in synovial fluid and paired serum is associated with disease activity and autoantibodies in RA. Clin Dev Immunol 2013; 2013:985301.

22 Mu R, Huang HQ, Li YH, Li C, Ye H, Li ZG. Elevated serum interleukin 33 is associated with autoantibody production in patients with rheumatoid arthritis. J Rheumatol 2010; 37:2006-2013.

23 Ali OS, Khalifa Al, Atia HA. Levels of interleukin 33 and interleukin 27 in rheumatoid arthritis and osteoarthritis in Egyptian patients. Int $\mathrm{J}$ Adv Biotechnol Res 2014; 15:465-473.

24 Hong YS, Moon SJ, Joo YB, Jeon CH, Cho ML, Ju JH, et al. Measurement of interleukin-33 (IL-33) and IL-33 receptors (SST2 and ST2L) in patients with rheumatoid arthritis. J Korean Med Sci 2011; 26:1132-1139.

25 Lee DM, Friend DS, Gurish MF, Benoist C, Mathis D, Brenner MB. Mast cells: a cellular link between autoantibodies and inflammatory arthritis. Science 2002; 297:1689-1692.

26 Xu D, Jiang HR, Li Y, Pushparaj PN, Kurowska-Stolarska M, Leung BP, et al. IL-33 exacerbates autoantibody-induced arthritis. J Immunol 2010; $184: 2620-2626$ 